\title{
Developing potato varieties with decreased cold-induced sweetening
}

Egorova A. ${ }^{1,2 *}$, Gerasimova S. ${ }^{1,2}$, Ibragimova S. ${ }^{1}$, Romanova A. ${ }^{1}$, Saboiev I. ${ }^{1}$, Domrachev D. ${ }^{5}$, Koeppel I. ${ }^{3}$, Hertig C. ${ }^{3}$, Hiekel S. ${ }^{3}$, Chamas S. ${ }^{3}$, Kumlehn J. ${ }^{3}$, Rogosina E. ${ }^{4}$, Chalaya N. ${ }^{4}$, Salina E. ${ }^{1}$, Kochetov A. ${ }^{1,2}$

${ }^{1}$ Kurchatov Genomic Center of the Institute of Cytology and Genetics, SB RAS, Novosibirsk, Russia

${ }^{2}$ Novisibirsk State University, Novosibirsk, Russia

${ }^{3}$ Leibniz Institute of Plant Genetics and Crop Plant Research, Gatersleben, Germany

${ }^{4}$ N.I. Vavilov All-Russian Institute of Plant Genetic Resources, St. Petersburg, Russia

${ }^{5}$ Institute of Organic Chemistry SB RAS, Novosibirsk, Russia

*email: egorova@bionet.nsc.ru

Commercial storage of potato at low temperatures $\left(4-6{ }^{\circ} \mathrm{C}\right)$ leads to the degradation of starch into reducing sugars, referred to as cold-induced sweetening (CIS). Upon processing at high-temperature, these sugars react with free amino acids, resulting in brown, bitter-tasting products. Vacuolar invertase breaks down sucrose to glucose and fructose, thereby largely contributing to the accumulation of reducing sugars. Sitedirected mutagenesis of the POTATO ACID INVERTASE 1 (PAIN1) is considered as a viable strategy towards the development of varieties with decreased cold-induced sweetening. To this end, cas $9 / \mathrm{gRNA}$ vectors were constructed for target sites in this gene. After having demonstrated the mutagenic activity of the vectors in potato protoplasts these constructs were delivered into cells of local potato varieties using biolistic DNA transfer. The regeneration of transformed plants is in progress. In addition, a second strategy involves the development of CIS-resistant donors for potato breeding from wild potato genotypes. Solanum chacoense is a wild relative of cultivated potato exhibiting strong CIS resistance. However, S. chacoense is toxic owing to high amounts of steroidal glycoalkaloids (SGAs). Consequently, the development of S. chacoense derivatives with much reduced toxicity may of great benefit for potato breeding programs. Knockout of the GLYCOALKALOID METABOLISM 9 (GAME9) gene, the regulator of SGA synthesis in potato, is considered as a useful principle to reduce SGAs in plants. Two $S$. chacoense accessions were selected from the VIR genebank collection as potential trait donors, for which low accumulation of reducing sugars and high accumulation of SGAs was shown. The cas $9 / \mathrm{gRNA}$ constructs used to knock out GAME9 were created and introduced into $S$. chacoense explants.

Acknowledgements: The study is supported by the RFBR (20-016-00217) and by the Kurchatov Genomic Centre of the Institute of Cytology and Genetics, SB RAS (075-152019-1662). 